\title{
Self administered cognitive screening test (TYM) for detection of Alzheimer's disease: cross sectional study
}

\author{
Jeremy Brown, consultant neurologist George Pengas, clinical research fellow Kate Dawson, research nurse \\ Lucy A Brown, honorary research assistant Philip Clatworthy, clinical research fellow
}

\author{
Department of Neurology, \\ Addenbrooke's Hospital, \\ Cambridge CB2 2QQ \\ Correspondence to: J Brown \\ jmb75@medschl.cam.ac.uk \\ Cite this as: BMJ 2009;338:b2030 \\ doi:10.1136/bmj.b2030
}

\section{ABSTRACT}

Objective To evaluate a cognitive test, the TYM ("test your memory"), in the detection of Alzheimer's disease.

Design Cross sectional study.

Setting Outpatient departments in three hospitals, including a memory clinic.

Participants 540 control participants aged 18-95 and 139 patients attending a memory clinic with dementia/ amnestic mild cognitive impairment.

Intervention Cognitive test designed to use minimal operator time and to be suitable for non-specialist use.

Main outcome measures Performance of normal controls on the TYM. Performance of patients with Alzheimer's disease on the TYM compared with age matched controls. Validation of the TYM with two standard tests (the minimental state examination (MMSE) and the Addenbrooke's cognitive examination-revised (ACE-R)). Sensitivity and specificity of the TYM in the detection of Alzheimer's disease.

Results Control participants completed the TYM with an average score of $47 / 50$. Patients with Alzheimer's disease scored an average of 33/50. The TYM score shows excellent correlation with the two standard tests. A score of $\leq 42 / 50$ had a sensitivity of $93 \%$ and specificity of $86 \%$ in the diagnosis of Alzheimer's disease. The TYM was more sensitive in detection of Alzheimer's disease than the minimental examination, detecting $93 \%$ of patients compared with $52 \%$ for the mini-mental state exxamination. The negative and positive predictive values of the TYM with the cut off of $\leq 42$ were $99 \%$ and $42 \%$ with a prevalence of Alzheimer's disease of $10 \%$. Thirty one patients with nonAlzheimer dementias scored an average of 39/50.

Conclusions The TYM can be completed quickly and accurately by normal controls. It is a powerful and valid screening test for the detection of Alzheimer's disease.

\section{INTRODUCTION}

Dementia and other cognitive problems are common. An estimated 24 million individuals in the world have dementia and the number affected will double every 20 years. $^{1}$ Milder forms of cognitive dysfunction, including mild cognitive impairment, affect many more people. ${ }^{2}$ Alzheimer's disease is the commonest form of dementia. Cognitive problems are a feature of many neurological and medical diseases including stroke, Parkinson's disease, head injury, and epilepsy.

Assessment of a patient's cognition is a crucial part of many medical consultations. Cognitive tests aid the diagnosis of dementia and are important in the medical and social management of patients and in the assessment of capacity. Once there are effective treatments for Alzheimer's disease there will be an even greater need for a quick sensitive test that is suitable for use in primary care and by non-specialists.

Many cognitive tests are available but none meets the three critical requirements for widespread use by a non-specialist - that is, take minimal operator time to administer, test a reasonable range of cognitive functions, and are sensitive to mild Alzheimer's disease. We designed the TYM ("test your memory") to fulfil these requirements. The paradox of thorough testing in minimal time was achieved by allowing patients to fill in the test themselves.

The TYM was administered to 540 normal controls aged 18-95, 108 patients with Alzheimer's disease/ amnestic mild cognitive impairment, and 31 patients with non-Alzheimer's degenerative dementias. The test was validated by comparing scores with those obtained with the mini-mental state examination ${ }^{3}$ and Addenbrooke's cognitive examination-revised. ${ }^{4}$ The Addenbrooke's revised test was developed from the original examination ${ }^{5}$ and is similar in both content and scoring. ${ }^{4}$ We determined the specificity and sensitivity of the TYM in the detection of Alzheimer's disease by comparing the scores of 94 patients with Alzheimer's disease with the scores of 282 age matched controls. Three scorers of differing backgrounds marked 100 tests to assess inter-rater reliability.

\section{METHODS}

The TYM test

The TYM is a series of 10 tasks on a double sided sheet of card with spaces for the patient to fill in (see appendix 1 on bmj.com). The patient's ability to complete the test is an 11th task. The tasks are orientation (10 points), ability to copy a sentence ( 2 points), semantic knowledge (3 points), calculation (4 points), verbal fluency ( 4 points), similarities ( 4 points), naming (5 points), visuospatial abilities ( 2 tasks, total 7 points), 
and recall of a copied sentence ( 6 points). The ability to do the test is also scored (5 points), giving a possible total of 50 points. The scores for the subsets are printed on the card and the total score calculated by adding the subset scores. To ensure consistent scoring a single sheet of scoring instructions is available.

\section{Participants}

\section{Selection of patients with Alzheimer's disease}

Patients were seen and diagnosed by a consultant neurologist with an interest in dementia in a dedicated memory clinic at Addenbrooke's Hospital. Patients attended the memory clinic between March and December 2007 and underwent neurological assessment, the Addenbrooke's cognitive examination-revised (which includes the minimental state examination), structural imaging, and blood tests. Many also had a psychiatric and neuropsychological assessment. The diagnosis of Alzheimer's disease was made with the NINCDS-ARDRA (National Institute of Neurological and Communicative Disorders and StrokeAlzheimer's Disease and Related Disorders Association) criteria for probable Alzheimer's ${ }^{6}$ without reference to the TYM result. The diagnosis of amnestic mild cognitive impairment was made according to published criteria. ${ }^{7}$ We excluded patients whose cognitive problems were thought to be substantially caused by depression.

A total of 108 patients (59 men, 49 women) received a clinical diagnosis of Alzheimer's disease or amnestic mild cognitive impairment. Alzheimer's disease was diagnosed in 85 and amnestic mild cognitive impairment in 23. Amnestic mild cognitive impairment has clinical and pathological similarities to Alzheimer's disease $^{89}$ and patients with amnestic mild cognitive impairment who score poorly on the Addenbrooke's cognitive examination are likely to progress to Alzheimer's disease. ${ }^{10}$ These patients were regarded as having early Alzheimer's disease. Nine patients with a diagnosis of amnestic mild cognitive impairment who scored below the cut off for dementia on the Addenbrooke's cognitive examination-revised $\left(\leq 83^{4}\right)$ were included in the Alzheimer's cohort. Patients with a diagnosis of amnestic mild cognitive impairment who score well on the Addenbrooke's cognitive examination are unlikely to progress to Alzheimer's disease over the next two years ${ }^{10}$; such patients might never progress to Alzheimer's disease and might return to normal. ${ }^{11}$ The 14 patients with a diagnosis of amnestic mild cognitive impairment who scored $>83$ on the ACE-R were analysed separately.

Table 1| Average scores on memory test (TYM), Addenbrooke's cognitive examination-revised (ACE-R), and mini-mental state examination (MMSE) and variability of these scores for patients with Alzheimer's disease $(n=94)$

\begin{tabular}{lc} 
& Mean (SD) raw score \\
TYM (total) & $33.2(8.2)$ \\
\hline ACE-R & $66.9(12.0)$ \\
\hline MMSE & $22.5(3.8)$ \\
\hline
\end{tabular}

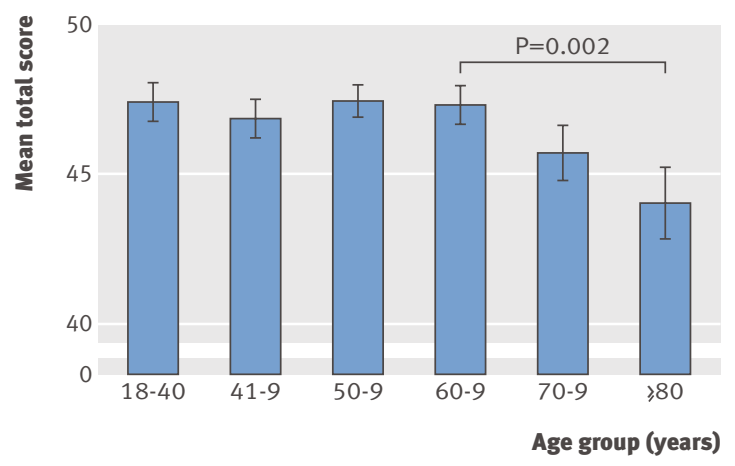

Fig 1| Mean total TYM scores (out of maximum possible score of 50) in control participants grouped according to age (decade). Errors bars are 2SE. Post-hoc testing showed significant impairment in performance for participants aged >80 compared with all younger age groups except age 71-80 (mean difference in score between age groups 61-70 and $>80$ was $2.7, \mathrm{P}=0.002$ )

\section{Selection of controls}

Controls were recruited from relatives accompanying patients to the memory clinic. Additional controls were recruited from relatives of patients attending neurology and medical outpatients departments at two other hospitals. We also included some dermatology outpatients. We excluded people with a history of neurological disease, memory problems, or brain injury. All participants gave informed consent.

We tested 540 controls aged $18-95$. Over half $(54 \%)$ were women. We calculated normal values for each decade from the age of 30 with standard errors. Three age matched controls were selected for each patient with Alzheimer's disease. To assess any differences in controls between different hospitals, we compared 100 controls from Addenbrooke's with 100 age matched controls from the other hospitals. To assess any sex differences, we compared 100 male controls with 100 age matched female controls.

\section{Testing and validation}

The 94 patients in the Alzheimer's cohort were given the TYM as well as the Addenbrooke's cognitive examination-revised (that includes the mini-mental state examination). The TYM was administered when the patient first arrived in the clinic.

We compared the scores of patients and age matched controls on subsets and total scores using $t$ tests with Bonferroni correction for multiple comparisons (equal variances not assumed). We compared the TYM scores of patients with the Addenbrooke's and mini-mental scores using Pearson's correlation coefficients. The internal consistency of the TYM was assessed with Cronbach's $\alpha$.

\section{Sensitivity and specificity of TYM in mild Alzheimer's} disease

We used data from the 94 patients with Alzheimer's disease to plot a receiver operating characteristic curve. We randomly selected three age matched controls $(\mathrm{n}=282)$ from the 540 controls for each patient with mild Alzheimer's disease. We calculated positive 
and negative predictive values for different TYM scores for different prevalences of Alzheimer's disease.

Sensitivity of TYM $v$ mini-mental state examination

A direct comparison between the TYM and the minimental state examination in identifying the 94 patients with Alzheimer's disease was performed by calculating the percentage of patients with Alzheimer's disease who were detected using the cut off of $\leq 42$ for the TYM and $\leq 23$ for the mini-mental state examination (the accepted cut off for dementia ${ }^{12}$ ).

Performance of TYM $v$ other tests in other forms of dementia and mild cognitive impairment

In the same period, 31 patients (17 men, 14 women; average age 63.3) with other degenerative dementias seen in the Addenbrooke's memory clinic as new patients were given the TYM and Addenbrooke's cognitive examination-revised. Of these patients, 16 had dementia with Lewy bodies, 13 had frontotemporal dementia, and two had progressive supranuclear palsy. The diagnoses were made according to accepted criteria. ${ }^{13-15}$ Pearson coefficients were used to calculate the correlation between the TYM scores and the other examinations.

TYM in mild cognitive impairment

Fourteen patients in the mild cognitive impairment cohort (average age 67.9) were given the TYM as well as the Addenbrooke's examination. We compared these scores with the TYM scores attained by the controls used for the Alzheimer's cohort.

Inter-rater variability

Three individuals scored the same 100 TYM sheets (38 patients, 62 controls) using the scoring sheet. One was a consultant experienced in the diagnosis of degenerative dementia (JB), one was a neurology specialist registrar working in the memory clinic (GP), and one a registered general nurse (LAB) with

\begin{tabular}{|c|c|c|c|c|}
\hline Subscore (maximum) & Controls & Patients & Difference & P value* \\
\hline Orientation (10) & $9.9(0.5)$ & $8.3(2.0)$ & 1.6 & $<0.001$ \\
\hline Copying (2) & $1.8(0.6)$ & $1.7(0.7)$ & 0.1 & 0.09 \\
\hline $\begin{array}{l}\text { Semantic knowledge } \\
\text { (3) }\end{array}$ & $2.6(0.6)$ & $1.4(1.0)$ & 1.3 & $<0.001$ \\
\hline Calculation (4) & $3.7(0.6)$ & $3.1(1.2)$ & 0.7 & $<0.001$ \\
\hline Fluency (4) & $3.5(1.0)$ & $2.2(1.5)$ & 1.4 & $<0.001$ \\
\hline Similarities (4) & $3.4(1.0)$ & $3.0(1.3)$ & 0.5 & 0.002 \\
\hline Naming (5) & $4.9(0.4)$ & $4.4(1.1)$ & 0.5 & $<0.001$ \\
\hline Visuospatial 1 (3) & $2.7(0.7)$ & $1.8(1.2)$ & 1.0 & $<0.001$ \\
\hline Visuospatial 2 (4) & $3.7(0.7)$ & $2.9(1.5)$ & 0.8 & $<0.001$ \\
\hline Anterograde (6) & $5.2(1.5)$ & $0.9(1.8)$ & 4.2 & $<0.001$ \\
\hline Executive (help) (5) & $5.0(0.2)$ & $3.7(1.2)$ & 1.3 & $<0.001$ \\
\hline Total (50) & $46.6(4.0)$ & $33.2(8.2)$ & 13.4 & $<0.001$ \\
\hline
\end{tabular}

*Two tailed significance (uncorrected). Significance values shown are for independent samples $t$ tests and are uncorrected; after Bonferroni correction for multiple comparisons level for significance is $\mathrm{P}=0.004$.

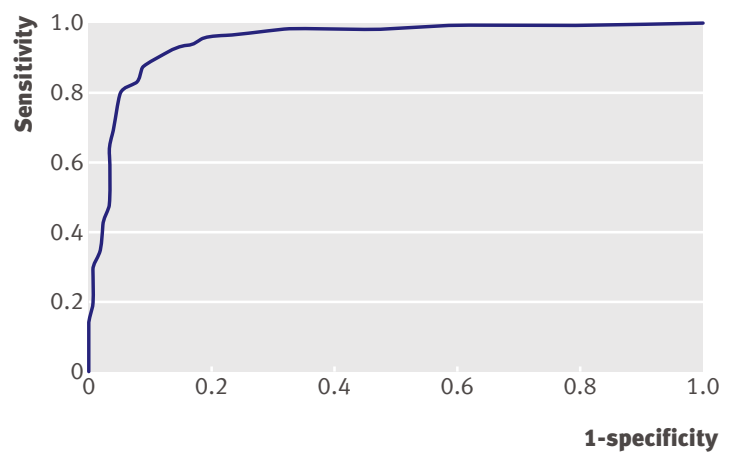

Fig 2 | Receiver operating characteristic curve for TYM scores differentiating between healthy controls $(n=282)$ and patients with Alzheimer's disease $(n=94)$

no specialised experience of patients with dementia who received 10 minutes of tuition. The tests were scored independently.

\section{RESULTS}

\section{Reliability and normative data}

The TYM was filled in quickly and efficiently by controls and patients with minimal supervision from a receptionist or nurse. The average time for a control to complete the test was five minutes.

The value of Cronbach's $\alpha$ for all participants and subsets was 0.80 . Figure 1 shows the results for control participants grouped by age. The average TYM score was about 47 for ages 18-70. Above the age of 70, there was a small decline in performance, which became significant above the age of 80 . One way analysis of variance confirmed a significant effect of age group on total TYM score $\left(F_{5,534}=6.4, \mathrm{P}<0.001\right)$ and showed a significant effect of age on the subscores: for copy $\left(F_{5,534}=5.4, \quad \mathrm{P}<0.001\right), \quad$ semantic $\quad$ knowledge $\left(F_{5,534}=3.7, \quad \mathrm{P}=0.003\right), \quad$ calculation $\quad\left(\mathrm{F}_{5,534}=3.1\right.$, $\mathrm{P}=0.008)$, fluency $\left(F_{5,534}=5.2, \mathrm{P}<0.001\right)$, similarities $\left(F_{5,534}=3.7, \quad \mathrm{P}=0.002\right), \quad$ anterograde memory $\left(F_{5,534}=3.5, \mathrm{P}<0.004\right)$, and help required $\left(F_{5,534}=8.0\right.$, $\mathrm{P}<0.001)$. All subscores except semantic knowledge showed a mild decrease with increasing age; semantic knowledge showed a small improvement.

We compared the performance of 100 controls from relatives in the memory clinic at Addenbrooke's with the performance of 100 age matched controls from the other hospitals. The mean age for both groups was 65 years $\left(t_{200}=0.04, \mathrm{P}=0.97\right)$. Mean TYM scores were similar in the two groups $\left(46.8 v 47.4, t_{200}=1.3, \mathrm{P}=0.19\right)$.

We examined the effect of sex on TYM scores by comparing the average scores for 100 men and 100 women in a cohort of age matched controls. Mean age for both groups was 61 years $\left(t_{200}=0.1, \mathrm{P}=0.92\right)$. The mean score for both groups was $47.4\left(t_{200}=0.05, \mathrm{P}=0.96\right)$.

\section{Alzheimer's disease and test validation}

Ninety four patients with Alzheimer's with an average age of 69.0 (SD 8.5) were tested with the TYM, the Addenbrooke's cognitive examination-revised, and the mini-mental state examination. Table 1 shows the average scores. 
Table $3 \mid$ Results of receiver operating characteristic analysis for detection of Alzheimer's disease with TYM in 94 patients with Alzheimer's and 282 age matched controls

\begin{tabular}{|c|c|c|c|c|c|c|c|c|c|c|c|c|c|c|}
\hline \multirow[b]{3}{*}{ Score } & \multirow[b]{3}{*}{ False +ve rate } & \multirow[b]{3}{*}{ Specificity (\%) } & \multirow[b]{3}{*}{ False-ve rate } & \multirow[b]{3}{*}{ Sensitivity (\%) } & \multicolumn{10}{|c|}{ Prevalence } \\
\hline & & & & & \multicolumn{2}{|c|}{$1 \%$} & \multicolumn{2}{|c|}{$5 \%$} & \multicolumn{2}{|c|}{$10 \%$} & \multicolumn{2}{|c|}{$20 \%$} & \multicolumn{2}{|c|}{$30 \%$} \\
\hline & & & & & PPV & NPV & PPV & NPV & PPV & NPV & PPV & NPV & PPV & NPV \\
\hline$\leq 38$ & 4 & 96 & 25 & 75 & 16 & 100 & 50 & 99 & 68 & 97 & 82 & 94 & 89 & 90 \\
\hline$\leq 39$ & 5 & 95 & 19 & 81 & 14 & 100 & 46 & 99 & 64 & 98 & 80 & 95 & 87 & 92 \\
\hline$\leq 40$ & 8 & 92 & 17 & 83 & 9 & 100 & 35 & 99 & 54 & 98 & 72 & 96 & 82 & 93 \\
\hline$\leq 41$ & 9 & 91 & 13 & 87 & 9 & 100 & 34 & 99 & 52 & 98 & 71 & 97 & 81 & 94 \\
\hline$\leq 42$ & 14 & 86 & 7 & 93 & 6 & 100 & 26 & 100 & 42 & 99 & 62 & 98 & 74 & 97 \\
\hline$\leq 43$ & 17 & 83 & 6 & 94 & 5 & 100 & 23 & 100 & 38 & 99 & 58 & 98 & 70 & 97 \\
\hline$\leq 44$ & 19 & 81 & 5 & 96 & 5 & 100 & 21 & 100 & 36 & 99 & 56 & 99 & 68 & 98 \\
\hline$\leq 45$ & 25 & 75 & 3 & 97 & 4 & 100 & 17 & 100 & 30 & 100 & 49 & 99 & 62 & 98 \\
\hline
\end{tabular}

$\mathrm{PPV}=$ positive predictive value; $\mathrm{NPV}=$ negative predictive value.

We found strong and significant correlations between all scores in patients with Alzheimer's (TYM v Addenbrooke's $\mathrm{R}^{2}=0.66, \mathrm{P}<0.001$; TYM v mini-mental $\mathrm{R}^{2}=0.51, \mathrm{P}=0.001$; Addenbrooke's $v$ mini-mental $\left.\mathrm{R}^{2}=0.70, \mathrm{P}<0.001\right)$. The Addenbrooke's examination contains the mini-mental state examination; the TYM has much less in common with the minimental or Addenbrooke's examinations.

Older patients with Alzheimer's (aged over 70) scored slightly better on the TYM than the younger patients with Alzheimer's, though the differences did not approach significance (average score $33.8 v 32.8$, $\left.t_{78}=0.57, \mathrm{P}=0.57\right)$.

\section{Comparison of TYM scores}

Table 2 shows comparisons between the total TYM score and subscores for patients and controls. The mean age was the same in both groups (69.0). As expected, patients with Alzheimer's were particularly impaired on anterograde memory scores relative to controls. Patients also scored poorly on semantic knowledge, fluency, visuospatial tasks, and executive function. After Bonferroni correction for multiple comparisons all subtests except sentence copying showed a significant decrease in patients with Alzheimer's compared with controls.

\section{Sensitivity and specificity of TYM}

The area under the ROC curve for differentiating between mild $\mathrm{AD}$ and controls was 0.95 (fig 2). If a single cut off is required then a TYM score of $\leq 42$ predicts mild Alzheimer's in this population, with a sensitivity of $93 \%$ and specificity of $86 \%$. Reducing the cut off to $\leq 39$ increases the specificity to $95 \%$, while raising the cut off to $\leq 44$ increases the sensitivity to $96 \%$.

Table 3 shows the negative and positive predictive value for various TYM scores at different prevalences of Alzheimer's. Negative predictive values are high: $100 \%$ for a score of $\leq 42$ with a prevalence of Alzheimer's up to $5 \%$. Positive predictive values are lower: a score of $\leq 42$ has a predictive value of $26 \%$ with a prevalence of Alzheimer's of 5\%.

\section{Comparison of sensitivity}

We compared the power of the TYM and the minimental state examination to detect the 94 patients with Alzheimer's. With a cut off of $\leq 42$ the TYM detected $93 \%$. With the established cut off of $\leq 23$ the mini-mental state examination detected $52 \%{ }^{12}$

TYM in non-Alzheimer's dementias

For the 31 patients with non-Alzheimer's dementia the mean TYM score was 38.9/50 (SD 8.6), the mean Addenbrooke's score was 77/100 (SD 16.0), and the mean mini-mental score was 25.3/30 (SD 4.1). The average mini-mental score is above the cut off for dementia, while the TYM and Addenbrooke's average scores are well below it, suggesting superior sensitivity of the TYM and the Addenbrooke's test in the diagnosis of non-Alzheimer's dementias.

The numbers with these individual diseases are too small to be meaningful, but there is a good correlation between the TYM scores of these 31 patients and the other scores (TYM v Addenbrooke's, Pearson's $\mathrm{R}^{2}=0.77$; TYM $v$ mini-mental, Pearson's $\mathrm{R}^{2}=0.74$; both $\mathrm{P}<0.001)$.

TYM in patients with mild cognitive impairment

The patients with mild cognitive impairment scored highly on all cognitive tests, averaging $27.8 / 30$ on the mini-mental state examination, 86.9 on the Addenbrooke's examination, and 45/50 on the TYM. Their scores on 10 of the subtests of TYM were similar to controls, but they tended to score worse than controls on anterograde memory $\left(3.4 / 6\right.$ v 5.2/6, $t_{13.5}=2.7, \mathrm{P}=0.02$ uncorrected).

\section{Inter-rater variability}

The TYM scores calculated by the three raters were highly correlated (table 4).

\section{DISCUSSION}

In this cross sectional study, the new "test your memory" test (TYM) was quick to use and detected $93 \%$ of cases of Alzheimer's disease. Control participants completed the TYM quickly and accurately. From 
the age of 18 to 70 the average score was stable at $47 /$ 50 , with a small decline after this age. Scores on all subsets of the TYM deteriorated with age, with the exception of semantic knowledge (these questions were designed for older patients).

Many of the controls were relatives of patients attending the memory clinic and came from the same population base. There was a slight male bias in the patients and slight female bias in the controls, but there was no significant difference in TYM scores between male and female controls. We recruited more controls from two other hospitals in East Anglia because the age range and number of the memory clinic controls was limited. The controls from the Cambridge memory clinic might have been expected to score slightly higher, but actually scored slightly lower. This effect was not large enough to be significant or to affect the results. Control participants were screened for a history of memory problems and neurological disease but not for other problems such as depression or vascular disease; such influences would be expected to reduce the TYM scores of controls and reduce differences in cognitive function between patients and control participants.

The TYM score of controls remained remarkably constant in widely different age ranges in both sexes and all geographical backgrounds. This is likely to be a ceiling effect. The TYM test was designed to be easy for normal controls to allow quick and accurate completion. Most controls of all ages scored almost full marks (for example, 68\% of controls aged 60-69 scored 48-50). This ceiling effect suggests that education and social class would have only mild effects on the TYM score, but we did not formally assess this. The reason for the low scoring controls was often apparent from the score sheet - lack of interest, reading problems, or a sense of humour. Some low scoring controls aged over 70 show a typical pattern for mild Alzheimer's and might be in the early stages (they were still included in the control group).

\section{Use in patients with Alzheimer's disease}

Patients with Alzheimer's disease performed much poorer than controls on the TYM. They scored an average of $33 / 50 ; 13.4$ points below the control group. Subtest analysis of scores in patients with Alzheimer's showed that all parts of the test, except copying a sentence, are performed less well by patients than controls. There is the expected pattern in patients with Alzheimer's performing poorly on tests of anterograde memory, semantic knowledge, fluency, and

Table $4 \mid$ Inter-rater agreement for memory test with three different raters, rating 100 randomly chosen participants ( 38 patients and 62 controls)

\begin{tabular}{lcccc} 
& Mean (SD) rawscore & \multicolumn{3}{c}{ Pearson's $r(r 2)$ correlation } \\
\cline { 3 - 5 } Rater & $(/ 50)$ & Consultant & Trainee & Nurse \\
Consultant & $43.8(6.4)$ & - & $0.99(0.98)$ & $0.99(0.98)$ \\
\hline Trainee & $43.8(6.5)$ & $0.99(0.98)$ & - & $0.99(0.98)$ \\
\hline Nurse & $43.8(6.5)$ & $0.99(0.98)$ & $0.99(0.98)$ & - \\
\hline
\end{tabular}

visuospatial tasks. They perform better (although still significantly worse than controls) on naming, orientation, similarities, and calculation subtests. Patients performed worse than controls in the copying subtest, but the difference was not significant; this test was probably too easy to identity patients with mild Alzheimer's.

We separately analysed patients with mild memory problems. These patients had a clinical diagnosis of amnestic mild cognitive impairment and scored well on the Addenbrooke's examination (>83). Amnestic mild cognitive impairment can be a prodrome of $\mathrm{Alz}^{-}$ heimer's, but some affected patients have mild problems that do not progress. ${ }^{11}$ The Addenbrooke's test is a good indicator of which patients with amnestic mild cognitive impairment (or questionable dementia) will develop progressive dementia in the two years after testing: patients who score $\leq 80$ are likely to progress, patients who score $>80$ are unlikely to progress. ${ }^{10}$ In this study as we used the higher cut off of $\leq 83$, the accepted cut off for dementia for the Addenbrooke's test, ${ }^{5}$ some non-progressive patients might have been included in the Alzheimer's cohort. The patients with mild cognitive impairment scored an average of $45 / 50$ on the TYM, with a trend towards problems in anterograde memory; they scored well in other subtests of the test.

Patients seen in the memory clinic in Cambridge could be different from those seen in other clinics. Cambridge attracts some younger patients with $\mathrm{Alz}$ heimer's from a wide area. The memory clinic serves a dual purpose in Cambridge, and many of the older patients in the study were local and referred directly from primary care. We separately examined the performance of the 42 patients with Alzheimer's aged 70 or over (average age 76.8). There was little difference in these patients compared with the younger patients either in overall TYM score (33.9 $v 32.6$ ) or in the subtest pattern. This suggests that it is a useful test to detect older patients with Alzheimer's.

The sensitivity and specificity of the TYM for detecting Alzheimer's in this cohort is high. A score of $\leq 42$ detects $93 \%$ of cases of mild Alzheimer's with a specificity of $86 \%$; a score of $\leq 44$ detects $96 \%$ of patients with mild Alzheimer's. The prevalence of dementia and mild cognitive problems (often with reduced insight) in the population sets a limit on the specificity of a test.

The testers and raters were usually aware of whether individuals were patients or controls at the time of testing or rating. As individuals fill in the test sheet themselves, the tester has minimal influence on the score. All scores were checked by a single rater (JB) using the scoring sheet. The use of a strict scoring scheme minimises the influence of the rater. Scores calculated by the single rater correlated closely with the scores of the other two raters in the inter-rater analysis.

\section{TYM $v$ mini-mental state examination}

The mini-mental state examination has been the standard short cognitive test for 30 years. It has proved valuable in the assessment of patients with established 


\section{WHAT IS ALREADY KNOWN}

There is no available short cognitive test that is quick to use, examines various skills, and is sensitive to Alzheimer's disease

There is confusion over how to score and interpret current short cognitive tests

\section{WHAT THIS PAPER ADDS}

The new "test your memory" (TYM) test is quick to use, examines 10 cognitive skills, and detects $93 \%$ of cases of Alzheimer's disease

There are normative data and a scoring sheet to allow consistent scoring and interpretation

dementia. It has many strengths but fails three of the requirements for a brief screening test for the non-specialist: minimal operator time, testing a wide range of cognitive domains, and sensitivity to mild Alzheimer's.

The mini-mental state examination takes an average of eight minutes to administer. Many clinicians find time to complete this but with an average consultation time in general practice in Europe of 10.7 minutes ${ }^{16}$ this is too long. Around 58\% of physicians in hospital practice thought the length of time taken to administer the mini-mental examination was too long. ${ }^{17}$

The mini-mental state examination tests several different cognitive domains but has a bias towards dominant parietal and temporal lobe function. It is a useful test of orientation, but the language and memory tests are too easy ${ }^{1819}$ and there is only a single point for visuospatial tasks. These drawbacks lead to the major problem with the test - that it is insensitive in the diagnosis of mild Alzheimer's. ${ }^{518-20}$ In community and hospital studies the sensitivity of the mini-mental state examination in detecting Alzheimer's using the established cut off of $\leq 23$ is low, varying from $49 \%$ to $69 \% .^{519-21}$ In our study it detected $52 \%$ of patients with Alzheimer's. This low detection rate probably reflects the high number of patients with mild Alzheimer's.

The TYM fulfils the three requirements. If a patient completes the test while in the waiting area supervised by the receptionist, it can be scored and analysed by the doctor in two minutes. If there is time during the consultation to observe the patients filling in the test, this can also be a useful aid to diagnosis.

The 11 TYM tasks examine more cognitive domains than the mini-mental state examination, with less bias towards dominant hemisphere language functions. The language and memory tests are more difficult. The inclusion of two visuospatial tasks, contributing $7 / 50$ points, is important in distinguishing Alzheimer's disease from pure amnestic syndromes. The test has a high sensitivity for detecting Alzheimer's. In our study it detected $93 \%$ of cases of Alzheimer's compared with $52 \%$ detected by the mini-mental state examination.

There are some additional problems with the minimental state examination. The score is influenced by how the tester asks the questions and also locationspatially disorientated patients might score 4 more points at home than in the clinic on the question of current location. The TYM is less influenced by the tester as it is filled in by the patient and will not vary with location.

The small range of scores in the mini-mental state examination limit its suitability for monitoring, but it is widely used for this purpose. It is the main test chosen by the National Institute for Health and Clinica Excellence (NICE) for deciding which patients in the United Kingdom should have cholinesterase inhibitors and for monitoring their response to treatment. ${ }^{22}$ The TYM has a much wider scoring range than the minimental state examination, with over 13 points between the average control and the average patient with mild Alzheimer's. In the group of 94 patients with Alzheimer's in this study the range of mini-mental scores was 14-30 while the range of TYM scores was 9-50 (2.5 times greater).

\section{TYM $v$ Addenbrooke's cognitive examination}

The original and revised Addenbrooke's examinations are sensitive and specific in the diagnosis of degenerative dementia. ${ }^{45}$ The major drawback of the revised examination is that it fails to fulfil the time requirement for a test for non-specialists, taking 20 minutes to administer and score. It tests a similar number of cognitive domains to the TYM and is sensitive to mild Alzheimer's.

There was a strong correlation between the memory score and the Addenbrooke's score in both Alzheimer's and non-Alzheimer's dementias. The TYM score averages $50 \%$ of Addenbrooke's score, enabling the two tests to be easily compared. Both tests provide a permanent record of the patient's performance, which can be assessed later.

TYM $v$ abbreviated mental test and other brief tests Several brief cognitive tests have been published for screening for memory problems. ${ }^{2324}$ Many of these are simple - such as clock drawing tests. These can be useful in distinguishing patients with established $\mathrm{Alz}_{-}$ heimer's from normal controls but are too limited for routine screening for mild Alzheimer's. ${ }^{25}$

The abbreviated mental test ${ }^{2627}$ is the established brief test. It fulfils the time requirement, taking only two minutes to administer. It does not test many cognitive domains and has a strong bias $(40 \%$ of the score) towards orientation, which is useful to detect delirium. ${ }^{28}$ It is less sensitive and specific than the mini-mental state examination in detecting mild Alzheimer's. ${ }^{29}$ There are additional problems with the abbreviated mental test, which is not given and scored consistently by regular users. ${ }^{30}$

\section{Other strengths and weaknesses of TYM}

The TYM has several other advantages over current bedside cognitive tests. There is a brief but rigorous scoring system. Inter-rater agreement for scoring is excellent. Ten minutes' training and the scoring sheet allowed a nurse, without experience of memory clinics, to score the TYM sheets as accurately as a specialist. 
The standardisation of the scoring should avoid the confusion that arises with scoring and interpreting the mini-mental state examination and the abbreviated mental test.

Cognitive testing usually depends on the tester and patient speaking the same language. The simplicity of the TYM should allow it to be administered and scored in a different language with help from a relative. It is currently being translated into four different languages. A minor adjustment to the semantic knowledge question (president for prime minister) is needed for American, Irish, and Australian use. Versions are being developed for UK patients who do not speak English as their first language; in these versions there are variations in the semantic knowledge and drawings to suit the background of the individual.

Normal values, sensitivity, and specificity are on the scoring sheet. As the test is filled in by the patient with little help and marked with the help of a scoring sheet, the tester and rater have little influence on the score.

The high negative predictive value of scores $\geq 42$ shows that in unselected groups a good score makes Alzheimer's disease unlikely and the TYM is a good screening test for memory problems. A score above 42 (that is, $\geq 43$ ) correctly excludes the diagnosis of Alzheimer's disease on $97 \%$ of occasions, even when the prevalence is as high as $30 \%$. In unselected groups the positive predictive value of a score of $\geq 42$ is relatively low, showing that the test alone cannot be used to diagnose Alzheimer's disease. In selected groups where the prevalence of Alzheimer's will be higher-for example, older patients with memory complaints - the positive predictive value of a score $\geq 42$ is much higher.

The TYM was used successfully in patients outside this study with more severe dementia, visual problems, or other physical disability. The tester needs to fill in the form with these patients and some tasks might be too difficult, so the scores of these patients are not comparable with scores of the patients or controls in this study. The test is also useful for patients with hearing impairment who have difficulty with verbal cognitive tests.

The TYM seems more sensitive than the mini-mental state examination in screening for non-Alzheimer's dementias. In the small group of such patients in our study the average mini-mental score was 25 , close to the average for the population. The TYM score in the same group was 39, eight points below the average control score.

A disadvantage of the memory test is the need for the specially printed sheets, though the Addenbrooke's cognitive examination-revised has a similar requirement. A website is being developed to help to solve this problem.

This work would not have been possible without the nurses and receptionists at Queen Elizabeth Hospital, King's Lynn, North Cambridgeshire Hospital, Wisbech, and Addenbrooke's Hospital, Cambridge, who administered the tests. We thank colleagues in the memory clinic, Cambridge, for permission to include their patients and for helpful discussions. A website is being prepared (www.tymtest.com) that will allow downloading of tests, scoring sheets, and instructions.
Contributors: JB devised and helped to design the TYM, he helped to perform and coordinated the clinical research, and wrote the first draft of the paper. GP helped in the clinical testing and in the writing of the paper and was an inter-rater tester. KD performed much of the clinical testing and scoring. PC performed the statistical analysis and prepared the figures and tables. LAB helped design the test and was an inter-rater tester. JB is guarantor.

Funding: GP was supported by Alzheimer's Research Trust (UK) and the Cambridge Commonwealth Trust. PC was supported by the Stroke Association. The authors are independent of any funders for this work. All authors had access to all data in this study.

Competing interests: None declared.

Ethical approval: This study was performed under ethical approval from Cambridgeshire 2 research ethics committee. All participants gave informed consent.

1 Ferri C, Prince M, Brayne C, Brodaty H, Fratiglioni L, Ganguli M, et al. Global prevalence of dementia: a Delphi consensus study. Lancet 2006;366:2112-7.

2 Graham JE, Rockwood K, Beattie BL, Eastwood R, Gauthier S, Tuokko H, et al. Prevalence and severity of cognitive impairment with and without dementia in an elderly population. Lancet 1997;349:1793-6.

3 Folstein MF, Folstein SE, McHugh PR. "Mini-mental state." A practical method for grading the cognitive state of patients for the clinician. J Psychiatr Res 1975;12:189-98.

4 Mioshi E, Dawson K, Mitchell J, Arnold R, Hodges J. The Addenbrooke's cognitive examination revised (ACE-R). A brief cognitive test battery for dementia screening. Int J Geriatr Psychiatry 2006;21:1078-85.

5 Mathuranath PS, Nestor PJ, Berrios GE, Rakowicz W, Hodges JR. A brief cognitive test battery to differentiate $A D$ and frontotemporal dementia. Neurology 2000;55:1613-20.

6 Mckhann G, Drachman D, Folstein M, Katzman R, Price D, Stadlan EM. Clinical diagnosis of AD. Neurology 1984;34:939-44.

7 Portet F, Ousset PJ, Visser PJ, Frisoni GB, Nobili F, Scheltens Ph, et al. Mild cognitive impairment $(\mathrm{MCl})$ in medical practice: a critical review of the concept and new diagnostic procedure. Report of the $\mathrm{MCl}$ Working Group of the European Consortium on Alzheimer's Disease. J Neurol Neurosurg Psychiatr 2006;77:714-8.

8 Morris JC, Storandt M, Miller P, McKeel D, Price J, Rubin E, Berg L. Mild cognitive impairment represents early-stage Alzheimer disease. Arch Neurol 2001;58:397-405.

9 Markesbery W, Schmitt F, Kryscio R, Davis D, Smith C, Wekstein D. Neuropathological substrate of mild cognitive impairment. Arch Neurol 2006;63:38-46.

10 Galton C, Erzinclioglu S, Sahakian BJ, Antoun N, Hodges JR. A comparison of the Addenbrooke's cognitive examination (ACE), conventional neuropsychological assessment, and simple MRIbased medial temporal lobe evaluation in the early diagnosis of Alzheimer's disease. Cog Behav Neurol 2005;18:144-50.

11 Ganguli M, Dodge HH, Shen C, DeKosky ST. Mild cognitive impairment, amnestic type. An epidemiological study. Neurology 2004;63:115-21.

12 Feher EP, Mahurin RK, Doody RS, Cooke N, Sims J, Pirozzolo FJ. Establishing the limits of the mini-mental state examination of subsets. Arch Neurol 1992;49:87-92

13 McKeith IG, Galasko D, Kosaka K, Perry EK, Dickson D, Hansen L, et al. Consensus guidelines for the clinical and pathological diagnosis of dementia with Lewy bodies (DLB). Report of the consortium on DLB international workshop. Neurology 1996;47:1113-24.

14 Neary D, Snowden JS, Gustafson L, Passant U, Stuss D, Black S, et al. Frontotemporal lobar degeneration: a consensus on clinical diagnostic criteria. Neurology 1998;51:1546-54.

15 Litvan I, Agid Y, Calne D, Campbell G, Dubois B, Duvoisin RC, et al. Clinical research criteria for the diagnosis of progressive supranuclear palsy (Steele-Richardson-Olszewski syndrome): report of the NINDS-SPSP international workshop. Neurology 1996;47:1-9.

16 Deveugele M, Derese A, van den Brink-Muinene A, Bensing J, De Maeseneer J. Consultation length in general practice: cross sectional study in six European countries. BMJ 2002;325:472.

17 Tangalos E, Smith G, Ivnik R, Petersen R, Kokmen E, Kurland L, et al. The mini-mental state examination in general medical practice: clinical utility and acceptance. Mayo Clin Proc 1996;71:829-37.

18 Naugle RI, Kawczak K. Limitations of the mini-mental state examination. Cleve Clin J Med 1989;56:277-81.

19 Galasko D, Klauber M, Hofstetter C, Salmon D, Lasker B, Thal L. The mini-mental state examination in the early diagnosis of AD. Arch Neurol 1990;47:49-52.

20 Tombaugh TN, McIntyre NJ. The mini-mental state examination: a comprehensive review. I Am Geriatr Soc 1992;40:922-35.

21 Bier J-C, Donckels V, Van Eyll E, Claes T, Slama H, Fery P, et al. The French Addenbrooke's cognitive examination is effective in detecting 
dementia in a French-speaking population. Dement Geriatr Cogn Disord 2005;19:15-7.

22 NICE technology guidelines 111. London: National Institute for Health and Clinical Excellence, 2007.

23 Lorentz WJ, Scanlan JM, Borson S. Brief screening tests for dementia. Can J Psychiatry 2002;47:723-32.

24 Woodford H, George J. Cognitive assessment in the elderly: a review of clinical methods. QJM 2007;100:469-84.

25 Philpot M. The clock-drawing test: a critique. Int Psychogeriatr 2004;16:251-6.

26 Hodkinson HM. Evaluation of a mental test score for assessment of mental impairment in the elderly. Age Ageing 1972;1:233-8.
27 Jitapunkul S, Pillay I, Ebrahim S. The abbreviated mental test: its use and validity. Age Ageing 1991;20:332-6.

$28 \mathrm{Ni}$ Chonchubhair A, Valacio R, Kelly J, O'Keefe S. Use of the abbreviated mental test to detect postoperative delirium in elderly people. Br J Anaesthesia 1995;75:481-2.

29 Mackenzie DM, Copp P, Shaw RJ, Goodwin GM. Brief cognitive screening of the elderly: a comparison of the mini-mental state examination (MMSE), abbreviated mental test (AMT) and mental status questionnaire (MSQ). Psychol Med 1996;26:427-30.

30 Holmes J, Gilbody S. Differences in use of abbreviated mental test score by geriatricians and psychiatrists. BMJ 1996;313:465.

Accepted: 6 February 2009 